\title{
Gaseous-Promotor-Assisted Direct Growth of Graphene on Insulating Substrates: Progress and Prospects
}

\author{
Ruojuan Liu ${ }^{1,2}$, Bingzhi Liu ${ }^{2,3}$, Jingyu Sun ${ }^{2,3, *}$, Zhongfan Liu 1,2,3, \\ ${ }^{1}$ Center for Nanochemistry, Beijing National Laboratory for Molecular Sciences, College of Chemistry and Molecular Engineering, \\ Peking University, Beijing 100871, China. \\ ${ }^{2}$ Beijing Graphene Institute (BGI), Beijing 100095, China. \\ ${ }^{3}$ College of Energy, Soochow Institute for Energy and Materials InnovationS (SIEMIS), SUDA-BGI Collaborative Innovation Center, \\ Soochow University, Suzhou 215006, Jiangsu Province, China.
}

\begin{abstract}
Utilizing a direct chemical vapor deposition approach to synthesize graphene on insulating substrates has received enormous attention to date in both scientific and technological realms. In contrast to the graphene growth on metal substrates, the catalytic inertness of insulators toward feedstock decomposition and the high energy barrier for carbon fragment migration on the insulating surface result in not only high density of grain boundaries but also a low growth rate. Thus-obtained graphene film is usually accompanied by massive defects and limited crystal quality, which adversely affect the physical integrity and electrical performance of the fabricated graphene-based device. In this respect, various strategies have been adopted to modify the direct growth processes of graphene, e.g., sacrificial metal catalysis approach, self-terminating confinement approach and near-equilibrium growth

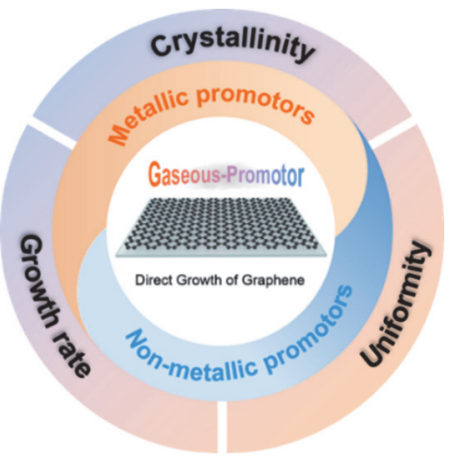
approach. Among these mentioned above, the gaseous-promotor-assisted growth methodology has proven to be a beneficial way in enhancing crystal quality and augmenting the growth rate of graphene. For the gaseous-promotorassisted chemical vapor deposition route, the gaseous promotor can not only regulate the composition/content of active carbon species in the gas-phase reaction process but also promote the surface migration and growth reactions. In this contribution, we review the recent advances in gaseous-promotor-assisted direct growth of graphene with high crystallinity, optimized uniformity, and enhanced growth rate on insulating substrates. First of all, we provide a systematic description of the growth behavior of graphene on insulators, including both the surface and gas-phase reactions combined with elementary steps during the growth process. We then summarize developed strategies aiming to achieve the direct growth of high-quality graphene via the assistance of gaseous promotors, with special emphasis on the effects and mechanisms of the growth process. The types of promotors commonly used in the gaseous-promotor-assisted strategy can be divided into metallic and non-metallic vapor species. These gaseous promotors can play influence on the feedstock decomposition, graphene nucleation, and enhance the enlargement and merging of individual domains. The corresponding mechanisms of the strategy can be classified into three parts: (1) The existence of highly concentrated metallic vapor species can promote thermal decomposition of carbon feedstock, which is the key to the growth of high-quality graphene; (2) The introduction of oxygen-containing species can effectively reduce the nucleation density, etch the amorphous carbon, leading to a high-quality, uniform growth of graphene film. In addition, hydroxylation of substrate through oxygen-containing species weakens the binding energy between the graphene edge and surface of the substrate, facilitating carbon fragment migration to evolve uniform monolayer graphene film; (3) The appearance of silicon and fluorine species reduces the growth kinetic barrier for carbon feedstock migrating onto the graphene edge to form the honeycomb lattice, which ensures
\end{abstract}

Received: November 4, 2021; Revised: November 27, 2021; Accepted: November 29, 2021; Published online: December 6, 2021.

*Corresponding authors. Emails: zfliu@pku.edu.cn (Z.L.); sunjy86@suda.edu.cn (J.S.).

The project was supported by the National Key R\&D Program of China (2019YFA0708201), the National Natural Science Foundation of China (61527814, 51702225), the Beijing National Laboratory for Molecular Sciences (BNLMS-CXTD-202001), and the Beijing Municipal Science \& Technology Commission (Z191100000819004).

国家重点研发计划项目(2019YFA0708201), 国家自然科学基金(61527814, 51702225), 北京分子科学国家研究中心(BNLMS-CXTD-202001)和北京市 科学技术委员会(Z191100000819004)资助

(C) Editorial office of Acta Physico-Chimica Sinica 
the ultrafast growth of graphene on insulating substrates. Finally, we describe existed challenges and present future perspectives on the direct growth of high-quality graphene on insulating substrates to stimulate more efforts devoted to direct graphene growth and ultimate applications. We hope this review can propel in-depth comprehension of the direct growth of graphene on insulators by gaseous-promotor-assisted strategy, and pave the way for the development and applications of graphene materials.

Key Words: Graphene; Chemical vapor deposition; Insulating substrate; Gaseous-promotor

\title{
气相助剂辅助绝缘祄底上石墨烯生长：现状与展望
}

\author{
刘若娟 1,2 , 刘冰之 ${ }^{2,3}$, 孙靖宇 $2,3,{ }^{*}$, 刘忠范 $1,2,3,{ }^{*}$ \\ 1 北京大学纳米化学研究中心, 北京分子科学国家研究中心, 北京大学化学与分子工程学院, 北京 100871 \\ 2 北京石墨烯研究院, 北京 100095 \\ 3 苏州大学能源学院, 苏州大学能源与材料创新研究院, 苏州大学-北京石墨烯研究院协同创新中心, 江苏苏州 215006
}

\begin{abstract}
摘要: 借助化学气相沉积(chemical vapor deposition, CVD)技术在绝缘祄底上直接生长的石墨烯薄膜, 在能源存储/转 换等领域有着广阔的应用前景。然而, 绝缘祄底表面石墨烯的生长呈现成核密度高、畴区尺寸小、生长速率低等特点, 获得的石墨烯薄膜往往具有较高的晶界密度和较低的层数均匀度, 严重制约着石墨烯基器件性能的发挥。在反应体系中 引入气相助剂可有效降低碳源裂解和石墨烯生长的能垒, 从而实现石墨烯品质与生长速率的提升。本文综述气相助剂辅 助绝缘祄底上石墨烯制备的方法: 首先对绝缘祄底上石墨烯的生长行为进行分析; 随后着重介绍几类常见的气相助剂辅 助石墨烯生长的策略和机理; 最后, 总结绝缘衬底上制备高品质石墨烯存在的挑战, 并对未来的发展方向进行展望。
\end{abstract}

关键词: 石墨烯; 化学气相沉积; 绝缘祄底; 气相助剂 中图分类号: 0647

\section{1 引言}

石墨烯是由 $s p^{2}$ 杂化碳原子键合形成的二维原 子晶体材料，具有高载流子迁移率 $\left(\sim 10^{5}\right.$ $\left.\mathrm{cm}^{2} \cdot \mathrm{V}^{-1} \cdot \mathrm{s}^{-1}\right)^{1}$ 、显著的室温霍尔效应 ${ }^{2}$ 、高透光性 $(\sim 97.7 \% \text {, 单层 })^{3}$ 、高机械强度(断裂应力 $130 \mathrm{GPa}$, 杨氏模量 $\sim 1.06 \mathrm{TPa}){ }^{4}$ 以及高热导率 $(\sim 5300$ $\left.\mathrm{W} \cdot \mathrm{m}^{-1} \cdot \mathrm{K}^{-1}\right)^{5}$ 等性质, 在传感 6 、能源存储 7 、光电子 器件 ${ }^{8-10}$ 以及智能材料 ${ }^{11-14}$ 等应用领域获得了广泛 关注。

制备决定未来, 实现高品质石墨烯材料的可 控制备是推动其走向产业化应用的关键。目前, 石 墨烯材料的制备方法包括机械 ${ }^{15}$ 或化学剥离 ${ }^{16,17}$ 、 碳化硅外延生长 ${ }^{18}$ 和化学气相沉积 (chemical vapor deposition, CVD) ${ }^{19}$ 等。其中, CVD法由于具有成 本低廉, 良好的可控性与可扩展性等特点, 是目前 制备高品质、大面积石墨烯薄膜的首选途径。该方 法通常选择具有催化活性的金属(例如铜、镍等)作 为石墨烯薄膜生长的祄底 ${ }^{20,21}$, 但在金属祄底表面 生长的石墨烯往往需要转移到目标祄底上(如石 英、蓝宝石、 $\mathrm{SiO}_{2} / \mathrm{Si}$ 等)以进行性能的表征和石墨 烯基器件的构筑等。然而, 工艺复杂的转移过程中
使用的溶剂和有机支撑材料等难免会导致石墨烯 的污染、褶皱与破损，进而影响石墨烯薄膜的各项 性能和物理完整性 22,23 而限制其应用。

因此, 在绝缘祄底上直接生长石墨烯薄膜可 有效规避转移过程带来的诸多问题并降低石墨烯 基器件的生产成本, 是促进其规模化生产和产业 化应用的重要途径。迄今为止, 已有大量研究报道 了不同种类绝缘祄底上石墨烯的 CVD制备, 如 $\mathrm{SiO}_{2} / \mathrm{Si}^{24,25}$ 、蓝宝石 ${ }^{26,27}$ 、六方氮化硼 28,29 、铁酸 锶 ${ }^{30}$ 、氮化硅 ${ }^{31}$ 和玻璃 $32-35$ 等。然而, 绝缘祄底极低 的碳源裂解催化活性以及在其表面碳物种高扩散 势垒, 导致石墨烯的成核和生长过程可控性差, 使 得绝缘祄底上制备的石墨烯成核密度高、畴区尺 寸小、生长速率低且层数均匀性差, 并伴随着高密 度的缺陷。

针对这些问题, 研究者们发展了反应动力学 调控 ${ }^{36-38}$ 、助剂辅助 $39-42$ 、等离子体辅助 $3-45$ 等多种 策略调控石墨烯的成核和生长行为。其中, 气相助 剂辅助法因可较好地介入气相反应过程和祄底表 面反应过程而被广泛研究。该方法通过调控反应 体系中碳物种的组分和含量、修饰祄底表面以促 
进活性碳物种迁移、降低边缘拼接势垒等方式, 成 功实现了对石墨烯成核及生长行为的有效调控。 在众多气相助剂中, 金属蒸气被证实具有催化碳 源裂解、促进石墨烯的边缘生长的积极作用, 因此 常被用作绝缘祄底上石墨烯生长的气相催化剂。除 金属蒸气外, 具有氧化刻蚀作用的含氧物质如氧 气、水、甲醇以及其他非金属类物质如氟化物、硅 烷和锗烷等也同样用于辅助绝缘祄底上石墨烯的 化学气相沉积直接生长。

本文将综述气相助剂辅助绝缘祄底上石墨烯 制备的策略。首先结合CVD法生长石墨烯的机理, 对绝缘祄底上石墨烯的生长行为进行分析; 随后 归纳并讨论几类常见的气相助剂辅助石墨烯生长 的方法和生长机制; 最后, 总结并展望在绝缘祄底 上制备高品质石墨烯面临的挑战和未来发展方向。

\section{2 绝缘祄底上石墨烯的生长行为}

在绝缘祄底上CVD法制备石墨烯薄膜过程 中, 由气态前驱体在祄底表面形成固态石墨烯的 反应历程十分复杂, 主要包含碳源裂解、活性碳物 种吸脱附与迁移、石墨烯成核与生长等基元步骤。 绝缘祄底的低溶碳性使得表面生长机制在石墨烯 生长中占主导地位 ${ }^{46,47}$ 。因此, 可将该生长过程分 为气相中发生的气相反应过程和祄底上发生的表 面反应过程两种 48 。对于气相反应过程, 主要的步 骤包括: 1)前驱体输运至CVD反应腔室; 2)气相前 驱体热裂解形成活性碳物种; 3)活性碳物种进一 步反应形成碳团簇以及副产物; 4)气相反应产物 吸附在祄底或石墨烯表面; 5)脱附的气相产物及 未反应完全的前驱体脱离反应腔室 ${ }^{49}$ 。对于绝缘祄 底上的表面反应过程, 主要的步骤包括: 1)碳前驱 体以及气相反应产物在绝缘祄底表面上的吸附; 2)活性碳物种在祄底表面的迁移和成核; 3)活性碳 物种直接结合到石墨烯核边缘生长为石墨烯畴 区；4)石墨烯畴区拼接形成连续的石墨烯薄膜。但 在实际的石墨烯生长过程中, 气相反应和表面反 应是相互关联的, 共同影响石墨烯薄膜的结晶质 量、层数等结构特性以及石墨烯的成核速率、生长 速率等动力学特性。

需要注意的是, 绝缘祄底表面大多是由具有 方向性和饱和性的共价键形成的非晶态结构, 这 类惰性祄底表面对碳前驱体的分解几乎没有催化 活性。因此, 与碳前驱体在金属衬底表面催化裂解 相比, 绝缘祄底上直接生长石墨烯往往需要更高 的生长温度 ${ }^{50}$ 或过饱和的表面碳物种浓度 ${ }^{51}$ 。然 而, 在此类生长条件下, 补底上方的气相粘滞层中
往往会聚集大量的碳物种或碳团簇 52 , 极易在反应 体系中形成影响石墨烯薄膜结晶质量的无定形碳 污染物 ${ }^{53}$ 。刘忠范课题组研究发现, 通过泡沫铜堆 垛 ${ }^{54}$ 或含铜碳源 ${ }^{55}$ 助催化法向 CVD体系中引入额 外的气相铜催化剂可调控气相反应过程, 利用其 提高气相反应中碳物种的催化裂解效率, 有效避 免了反应体系中无定形碳副产物形成, 从而实现 了较高品质石墨烯的可控制备。相应地, 该方法在 绝缘祄底上生长石墨烯过程中的可行性和有效性 同样得到验证 ${ }^{56}$ 。其次, 碳物种与绝缘祄底表面具 有较大的结合能 57,58 , 一方面会大大降低成核势 垒, 使祄底表面呈现出成核密度高的特点; 另一方 面会提高碳物种在表面的迁移势垒 ${ }^{59,60}$ (约为金属 衬底上的20倍)、导致祄底表面石墨烯的单畴生长 速率低( 1-3 nm· $\left.\mathrm{min}^{-1}\right)$ 。此外, 研究表明在金属祄 底上生长石墨烯的过程中, 氧气对石墨烯的刻蚀 作用可有效调控成核密度 ${ }^{61}$, 而对石墨烯生长边缘 的活化作用可提高生长速率 61,62 。鉴于此, 将氧气 或含氧化合物等气相助剂引入绝缘祄底石墨烯的 生长中, 同样实现了祄底表面石墨烯成核、生长等 表面反应过程的有效调控 63,64 。同时, 气相助剂有 望降低边缘拼接势垒 ${ }^{65}$ 和表面羟基化修饰祄底以 促进活性碳物种的迁移 ${ }^{66}$, 从而促进边缘生长、提 高石墨烯的直接生长速率。

目前, 对于绝缘祄底上石墨烯的生长, 气相助 剂能较好地介入气相反应过程和祄底表面反应过 程: 既可以通过促进碳前驱体裂解, 实现气相反应 过程中活性碳物种组分和含量的调控; 又可以通过 修饰衬底表面促进活性碳物种迁移、降低碳原子边 缘拼接势垒等方式调控祄底表面石墨烯的生长行 为。接下来将具体介绍金属和非金属气相助剂辅助 绝缘祄底上石墨烯的制备策略以及相对应的机制。

\section{3 金属气相助剂辅助石墨烯生长}

与热裂解相比, 金属的催化作用可有效降低 碳前驱体裂解反应能垒, 提高裂解效率, 进而在较 低的温度下得到裂解充分的碳物种, 有利于石墨 烯薄膜的品质提升和能耗的降低。在绝缘祄底表 面石墨烯的制备中, 金属催化辅助技术路线主要 分为牺牲金属镀层法和金属气相助剂法。其中, 牺 牲金属镀层法依靠预先在目标祄底表面沉积的金 属层促进碳源的裂解和石墨烯的生长 ${ }^{39,67}$ 。尽管牺 牲金属镀层法在一定程度上实现了绝缘祄底上高 质量石墨烯的制备, 但后续镀层去除过程中使用 的刻蚀剂、溶剂以及金属的残留都会引入污染, 从 而影响石墨烯薄膜的性能40。金属气相助剂法, 通 
常将金属簿或蒸镀的金属层置于气路上游或绝缘 祄底周围，利用高温下挥发产生的金属蒸气催化 裂解碳源, 促进石墨烯成核及生长。相应地, 该方 法由于催化剂悬浮在气相中, 与祄底不存在直接 物理接触, 有效克服了镀层去除过程带来的弊端。 金属蒸气的来源有多种, 根据金属源的形态可以 分为金属箔、液态金属和含金属化合物。

\section{1 金属箔}

铜的熔点相对较低, 在CVD生长石墨烯的温 度下易升华,因此, 铜箔常被用作金属蒸气源。Chiu 课题组 ${ }^{41}$ 在沿着气流方向的上游合适位置处放置 一块铜箔, 利用蒸发的铜蒸气催化甲烷裂解, 得到 的碳物种在下游 $\mathrm{SiO}_{2} / \mathrm{Si}$ 目标祄底表面进行石墨化 反应, 最终得到石墨烯薄膜(图1a)。该方法辅助制 备的石墨烯薄膜的拉曼表征结果如图 $1 \mathrm{~b}, \mathrm{c}$ 所示, 表明其结晶质量与绝缘祄底和铜䈃间的距离存在 依赖关系。这是因为连续释放的铜蒸气不断随气流 向下游迁移, 反应体系中不同位置的铜蒸气存在一 定程度的浓度差, 进而影响绝缘祄底上石墨烯制备 的均匀性。在此基础上, Choi课题组 ${ }^{68}$ 利用图 $1 \mathrm{~d}$ 展 示的策略将铜䈋无物理接触地悬挂在目标祄底上 方, 使绝缘祄底上方的铜蒸气浓度尽可能充足且均 匀, 从而制备出了无缺陷峰的高质量单层石墨烯薄 膜(图1e), 并且可实现石墨烯薄膜生长速率的提 升 ${ }^{69-71}$ 。进而, 该课题组 ${ }^{72}$ 采用一种三明治结构(图 1f), 将铜䈃置于目标石英祄底与 $\mathrm{SiO}_{2} / \mathrm{Si}$ 祄底间, 在 一定的温度下 $\left(>802{ }^{\circ} \mathrm{C}\right)$ 铜簿与 $\mathrm{SiO}_{2} / \mathrm{Si}$ 祄底形成液
态铜硅合金, 可获得高浓度具有催化作用的铜蒸 气。与悬空铜䈃的方法相比, 这种方法有望实现大 面积、高品质石墨烯的均匀制备(图1g)。此外, Chueh课题组 ${ }^{73}$ 在 $850{ }^{\circ} \mathrm{C}$ 的生长温度下, 利用置于 石英管内气流方向上下游的铜箔向反应体系中引 入铜蒸气, 成功实现将绝缘祄底表面预先沉积的无 定型碳膜催化转化为结晶态石墨烯。

\section{2 液态金属}

除金属铜之外, 金属镓因具有类似的低溶碳 性也受到研究者的广泛关注。在 $1000^{\circ} \mathrm{C}$ 时, 镓的 饱和蒸气压比铜高一个数量级, 即气相中会存在 更多的金属蒸气参与碳前驱体的催化裂解反应, 有 望提高裂解效率和石墨烯生长速率。Fujita课题组 ${ }^{42}$ 将镓液滴置于沿气流方向的上游, 通过释放蒸气 介入石墨烯生长的气相反应(图2a); 为了进一步提 高镓蒸气的浓度, 他们还将目标祄底覆盖在盛放 液态镓的氧化铝舟上, 实现了绝缘祄底上石墨烯 的制备(图 $2 b, c)$ 。付磊课题组 ${ }^{74}$ 发展了镓蒸气辅助 催化直接制备均匀单层石墨烯的策略, 并探索了 其在除雾方面的应用(图2d)。相较于转移法制备的 石墨烯除雾器, 直接生长石墨烯衍生的除雾器由 于石墨烯与祄底较强的界面结合作用(图2f-i), 可 有效阻止水分子的渗入, 在除雾时间(32 V电压下 仅需 $5 \mathrm{~s}$ )和稳定性方面都呈现出明显优势(图2e)。 此外, 于贵课题组 ${ }^{75}$ 将液态镓作为催化剂滴在祄底 表面, 成功在氮化硅祄底与镓液滴的界面制备出 了多层石墨烯薄膜。

(b)

(a)

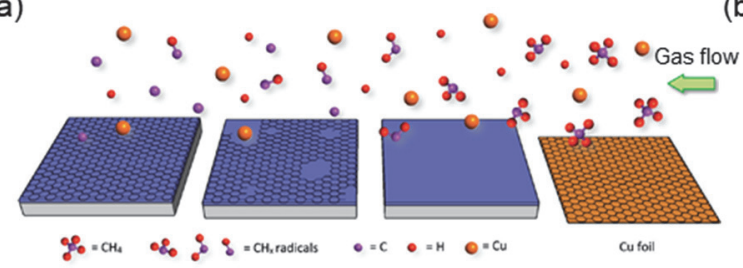

(d)

(e)

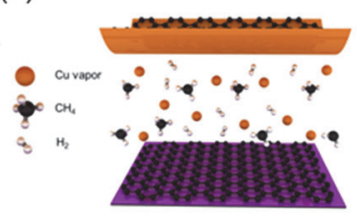

(c)
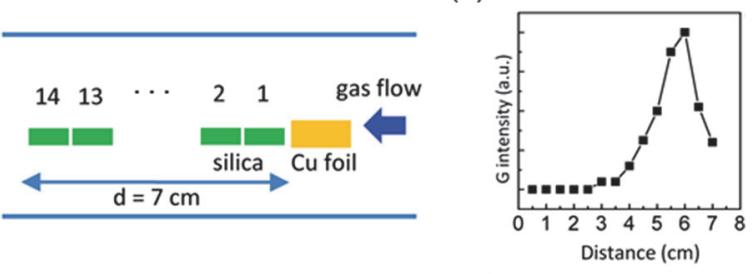

(g)

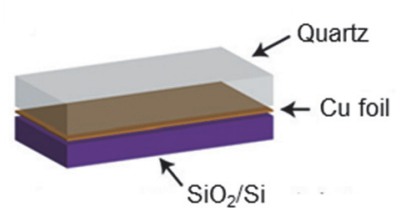

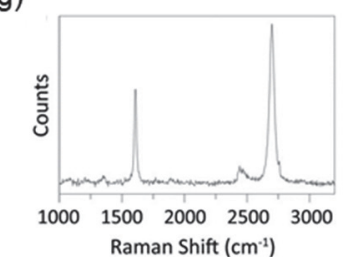

图 1 金属铜箔辅助石墨烯生长 ${ }^{41,68,72}$

Fig. 1 Copper-vapor sublimed from copper foil assisted the growth of graphene on insulating substrates ${ }^{41,68,72}$.

(a) Schematic of the distance-dependent graphene growth along the gas flow direction; (b) Growth setup; (c) The Raman G band intensity as a function of distance; (d) A schematic of the Cu-vapor-catalyst-assisted growth of graphene on quartz glass and $\mathrm{SiO}_{2} / \mathrm{Si}$ substrate, where the copper foil was laid over the substrate without physical contact; (e) The Raman spectrum of graphene grown on glass with the Cu-vapor catalyst; (f) Illustration of graphene growth on insulating substrates by physical contact of copper foil through the formation of Cu-Si alloy; (g) Raman spectrum obtained from a random position on quartz glass. (a-e) Adapted from American Chemical Society publisher; (f, g) Adapted from Wiley-VCH publisher. 
(a)

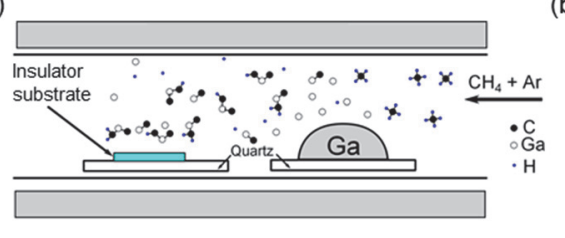

(d)

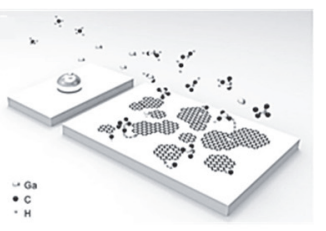

(b)

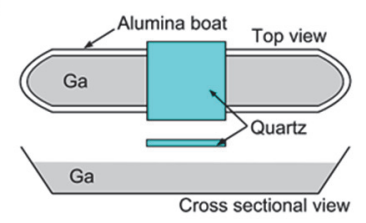

(f) (c)

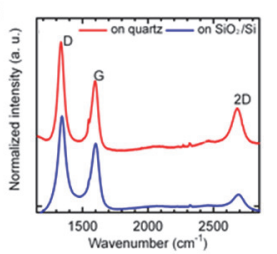

(g) (e)

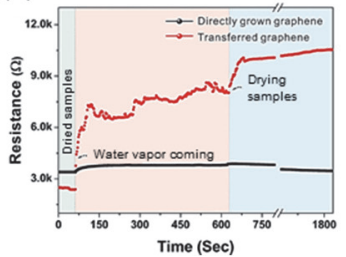

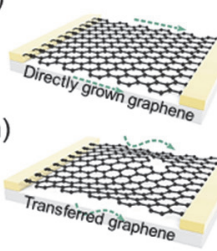

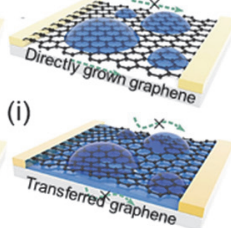

图 2 液态金属镓辅助石墨烯生长 ${ }^{42,74}$

Fig. 2 Graphene growth catalyzed by liquid metal Ga on the insulating substrate ${ }^{42,74}$.

(a) and (b) Schematic of two experimental setups for CVD using Ga vapor catalysts; (c) Typical Raman spectra of the graphene layers synthesized on quartz and $\mathrm{SiO}_{2} / \mathrm{Si}$ substrates. The inset is a SEM image of the graphene on quartz; (d) Schematic of direct growth of graphene on quartz with the assistance of

Ga vapor for transparent defogger; (e) The resistance profiles of directly grown graphene defoggers and transferred graphene defoggers before and after exposure to fog; (f) and (g) Upper surface of directly grown graphene absorbed by water molecules, while the current carriers passing through the under surface protected by the quartz substrate; (h) and (i) Both sides of transferred graphene absorbed by water molecules for lack of strong interaction force between the graphene and the quartz substrate. (a-c) Adapted from American Institute of Physics publisher; (d-i) Adapted from Wiley-VCH publisher.

(a)

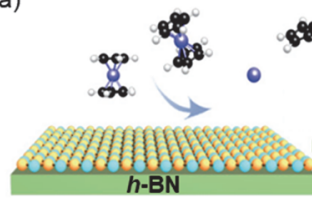

(c)

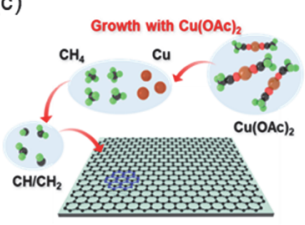

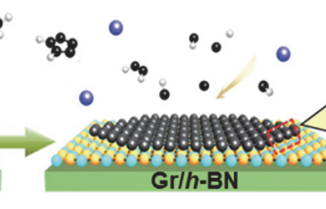

(d)

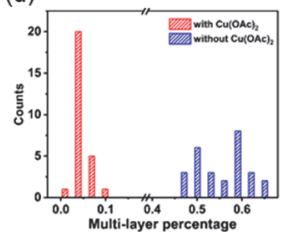

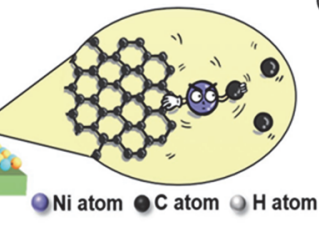

(e)

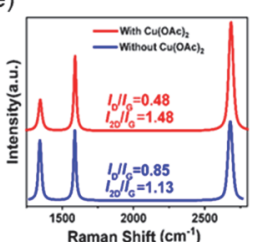

(b)

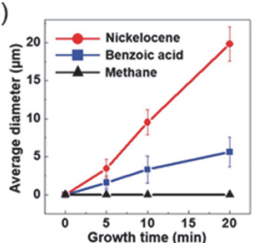

(f)

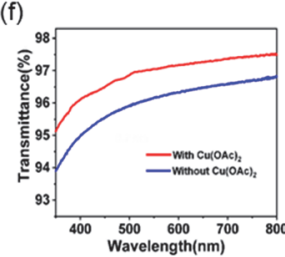

图 3 含金属化合物辅助石墨烯生长 ${ }^{56,76}$

Fig. 3 Metal-containing species assisted direct growth of graphene ${ }^{56,76}$.

(a) Illustration of graphene growth on $\mathrm{h}-\mathrm{BN} / \mathrm{Cu}$ foils using a nickelocene precursor; (b) Plot of the graphene domain size as a function of growth time using nickelocene (red), benzoic acid (blue), and methane (black) as precursors, respectively; (c) Schematic illustration of the graphene growth process with the presence of $\mathrm{Cu}(\mathrm{OAc})_{2} ;$ (d) Multilayer percentage of graphene grown with/without $\mathrm{Cu}(\mathrm{OAc})_{2}$; (e) Representative Raman spectra of graphene grown through the two routes; (f) Corresponding UV-Vis transmittance spectra of graphene films. (a, b) Adapted from Wiley-VCH publisher; (c-f) Adapted from Oxford University Press publisher.

\section{3 含金属化合物}

刘忠范课题组 ${ }^{76}$ 选取二茂镍作为石墨烯生长 的前驱体, 实现了大规模、高品质石墨烯/氮化硼 层间异质结的可控制备和快速生长。如图3a所示, 在 $150{ }^{\circ} \mathrm{C}$ 温度下, 二茂镍裂解为镍原子和碳五元 环, 可作为石墨烯生长的气相催化剂和碳物种; 以 其作前驱体在 $\mathrm{h}-\mathrm{BN} / \mathrm{Cu}$ 祄底上生长石墨烯的速率 约为苯甲酸作前驱体的四倍(图3b)。这是因为二茂 镍裂解产生的镍原子吸附到石墨烯生长的边缘,
显著降低了生长能垒, 提高了反应速率。

在此基础上, 刘忠范课题组 ${ }^{56}$ 采用醋酸铜辅助 法在蓝宝石祄底上制备了高结晶质量、低缺陷密 度的石墨烯薄膜(图3c)。通常地, 碳前驱体进入反 应体系后裂解得到的活性碳物种会在气相中通过 发生自由基链式反应等方式形成含碳原子数更多 的碳氢物种以及碳团簇, 进而吸附在石墨烯缺陷 位点产生无定形碳污染物。在蓝宝石祄底上无醋 酸铜辅助法生长石墨烯过程中, $\mathrm{CH}_{4}$ 热裂解为 $\mathrm{CH}_{3}$ 
后进一步裂解为 $\mathrm{CH}_{2}$ 的过程需克服较高能垒, 气相 中的碳物种得不到充分裂解, 会更容易形成分子 量较大的碳氢物种或碳团簇, 形成的无定形碳严 重影响石墨烯薄膜的质量。而醋酸铜分解提供的 铜团簇会促进碳物种的催化裂解, 避免气相反应 中形成大的碳氢物种或碳团簇, 从而抑制倾向于 作为多层核成核位点的无定形碳的形成, 最终提 高石墨烯薄膜的层数均匀性 77 。通过SEM表征结果 对多层石墨烯区域占比进行统计分析, 无醋酸铜 辅助法得到的石墨烯薄膜多层区域占比值在 $45 \%-$ $65 \%$ 之间,而醋酸铜辅助法生长的石墨烯薄膜的多 层区域占比值可降至 $10 \%$ 以内(图3d)。这充分表明 了醋酸铜辅助对多层石墨烯生长存在抑制作用, 制 备的石墨烯薄膜具有更高的结晶品质(图3e, f)。

\section{4 非金属气相助剂辅助石墨烯生长}

除金属助剂外, 含氧非金属气相助剂如氧气、
水、醇类物质以及其他非金属物质如氟化物、锗 烷、硅烷等同样被用于辅助绝缘祄底上石墨烯的 直接生长, 这种策略从根本上规避了金属残留的 问题。

\section{1 氧气}

刘云圻课题组 ${ }^{63}$ 提出一种氧气辅助石墨烯生 长的方法, 即在 $800^{\circ} \mathrm{C}$ 温度下, 将 $\mathrm{SiO}_{2} / \mathrm{Si}$ 祄底和石 英祄底置于空气氛围中退火 $1 \mathrm{~h}$ 后, 再升至 $1100^{\circ} \mathrm{C}$ 进行石墨烯生长(图4a)。空气氛围退火既可有效除 去祄底表面杂质，又可以通过氧修饰祄底表面增 强碳物种在祄底表面的吸附, 有利于石墨烯的成 核(图4b-d)。此外, 有研究表明氧气对石墨烯边缘 的刻蚀作用使得其生长边缘能保持化学活性 ${ }^{78}$, 能 通过降低石墨烯边缘生长的能垒促进已存在的石 墨烯畴长大。在此基础上, 刘忠范课题组 ${ }^{79}$ 在 CVD 法生长石墨烯的过程中持续引入痕量氧气, 成功 实现了耐高温石英祄底上高品质石墨烯的制备。其

(a)

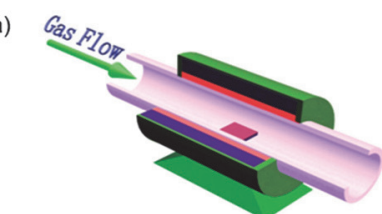

(b)
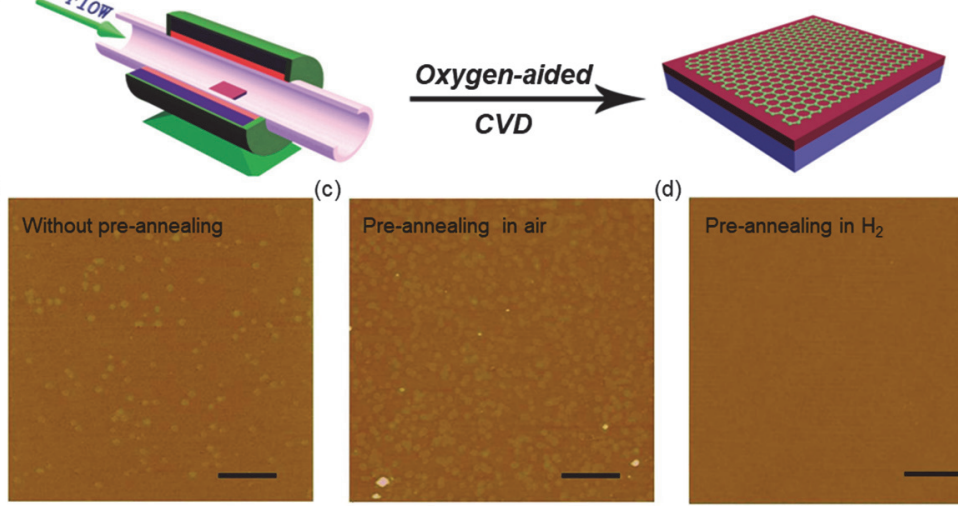

(e)

(g)
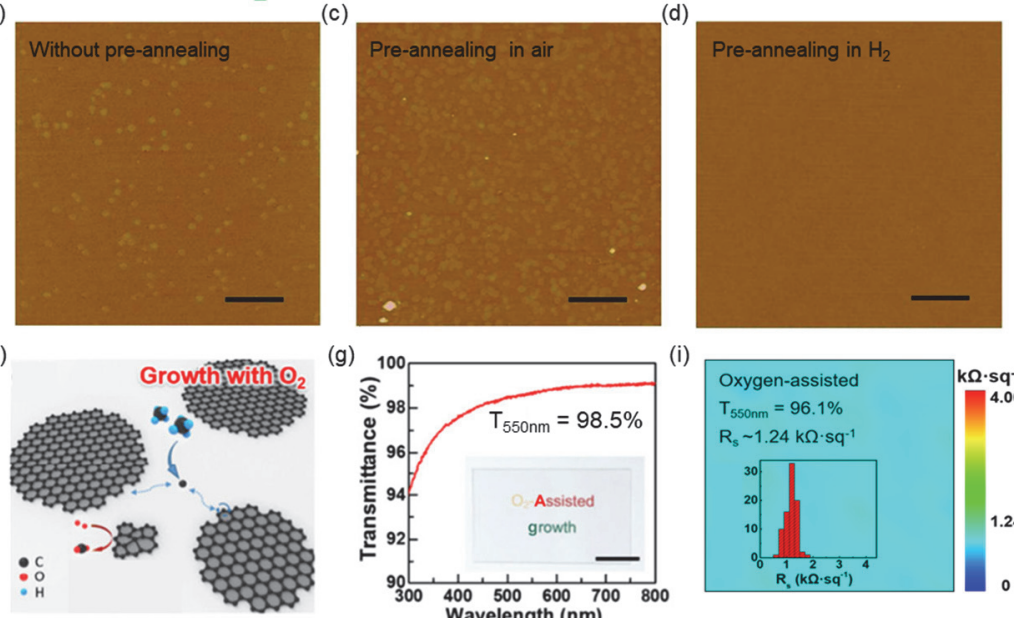

(f)
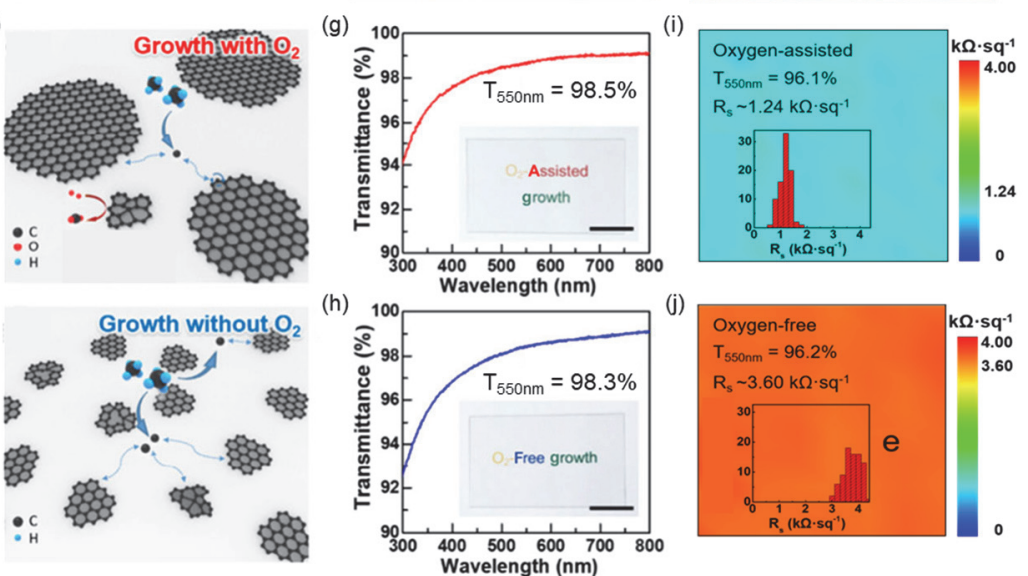

图 4 氧气辅助石墨烯生长 63,79

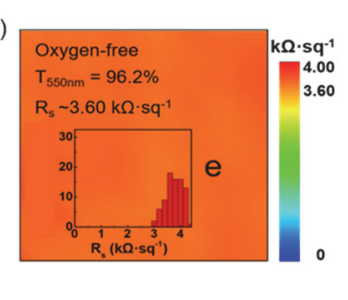

Fig. 4 Oxygen-aided growth of graphene on the insulating substrate ${ }^{63,79}$.

(a) Schematic diagram of the oxygen-aided CVD growth of graphene on $\mathrm{SiO}_{2} / \mathrm{Si}$ substrate; (b) Morphology of graphene without annealing treatment;

(c) Morphology of graphene grown after a pre-annealing treatment in air; (d) Morphology of graphene grown after a pre-annealing treatment in $\mathrm{H}_{2}$,

Scale bar $1 \mu \mathrm{m}$; (e) and (f) Schematic diagrams showing the surface reaction pathways for direct graphene growth on quartz throughout the $\mathrm{O}_{2}$-assisted and

$\mathrm{O}_{2}$-free CVD; (g) and (h) Optical transmittance spectra, (i) and (j) Spatial sheet resistance mapping of the two types of graphene glass, respectively. 
中, 改变氧气通入量可实现石墨烯成核密度的有 效调控; 适量的氧气有助于刻蚀能量不稳定的新 核, 降低石墨烯的成核密度, 提高石墨烯的畴区尺 寸(图4e, f); 而过量的氧气会加剧刻蚀作用, 导致 石墨烯畴区尺寸不增反减。由于石墨烯的电学输 运性质严重受到晶界密度的影响 ${ }^{80}$, 相比石英祄底 上常规CVD直接生长的石墨烯, 痕量氧气辅助法 将制备的石墨烯畴区从 $200 \mathrm{~nm}$ 提升至 $1.8 \mu \mathrm{m}$ 。如图 $4 \mathrm{~g}-\mathrm{j}$ 所示, 通过对比研究畴区大小与石墨烯薄膜导 电性能的关系, 发现将石英祄底上制备的石墨烯 畴区从百纳米级别提升至微米级别时, 在相同的 透过率下 $(\sim 96 \%, 550 \mathrm{~nm})$, 相比于普通CVD法制 备石墨烯玻璃 $\left(\sim 3.60 \mathrm{k} \Omega \cdot \mathrm{sq}^{-1}\right)$, 利用 $\mathrm{O}_{2}$ 辅助CVD法 制备的石墨烯玻璃具备明显下降的面电阻( 1.24 $\left.\mathrm{k} \Omega \cdot \mathrm{sq}^{-1}\right)$, 极大拓宽了石墨烯玻璃在透明加热和光 电探测领域的应用前景。

\section{2 含氧化合物}

2019年, 任文才课题组 ${ }^{64}$ 在 $\mathrm{SiO}_{2} / \mathrm{Si}$ 祄底上生长 石墨烯的过程中引入微量水, 实现了石墨烯薄膜 的快速均匀制备(图5a)。究其原因是水具有弱氧化 性且能促进祄底释放出氧, 这既可有效刻蚀反应 体系中的无定形碳以及薄膜中缺陷结构 (图 $5 \mathrm{~b}, \mathrm{c}$ ), 又可降低石墨烯边缘生长势垒(图5d), 提升石墨烯 制备的均匀性和生长速率。在此基础上, 刘忠范课 题组 ${ }^{81}$ 发展了一种利用水辅助制备石墨烯玻璃的 策略(图5e)。即高温生长条件下, 通过在CVD反应 室中引入微量水, 使其能够刻蚀体系中的无定形 碳以及能量不稳定的石墨烯核, 从而减少无定形 碳污染、降低成核密度、增大畴区尺寸(图5f)。制 备的石墨烯玻璃面电阻值仅为无水辅助的 $20 \%$, 有 效提高了石墨烯的透明导电性能。此外, 于贵课题 组 ${ }^{82}$ 提出一种前驱体调控生长的策略。他们在前驱 体中加入甲醇, 利用其高温分解产生微量的水修 饰衬底表面, 使 $\mathrm{SiO}_{2}$ 表面转变为羟基终止, 从而促 进石墨烯初级形核的边缘生长、抑制其二次成核, 实现了初级成核主导的单层石墨烯控制制备(图 $5 \mathrm{~g}-\mathrm{i})$ 。理论计算表明, 石墨烯纳米带边缘碳原子与 硅原子表面的结合能高于其与羟基终止表面的结 (a)

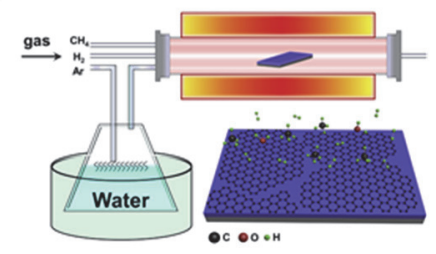

(b)

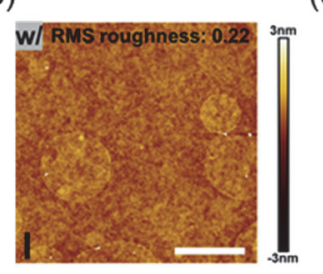

(c)

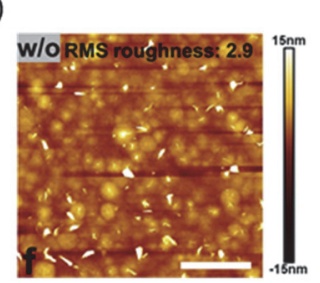

(d)

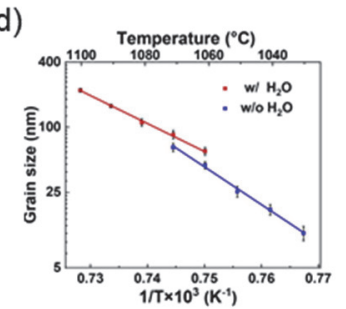

(e)

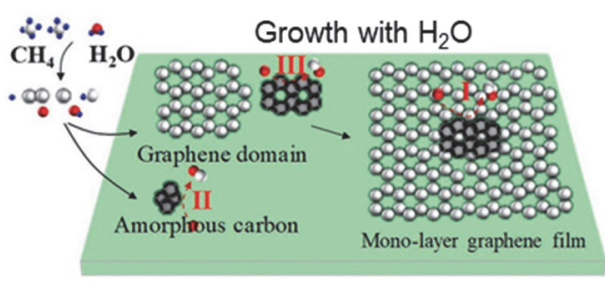

(g)

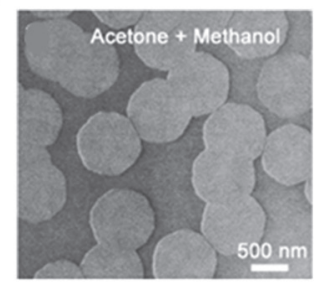

(h)

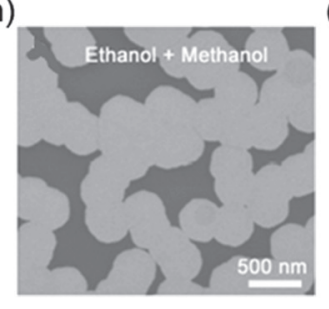

(f)

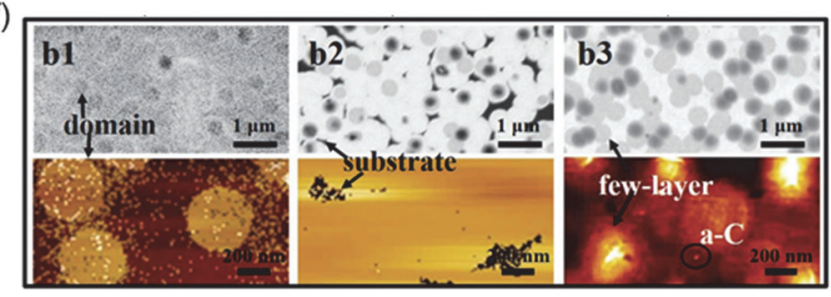

(i)
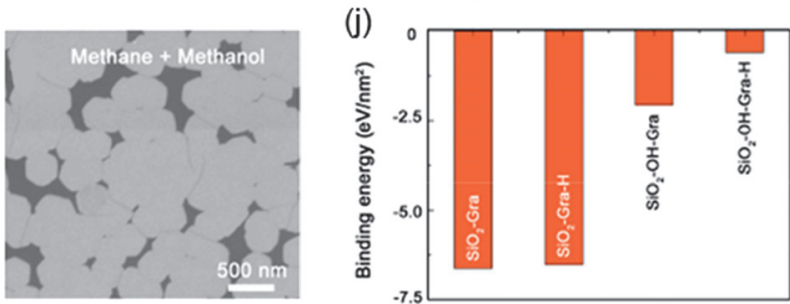

图 5 含氧化合物辅助石墨烯生长 ${ }^{64,81,82}$

Fig. 5 Oxygen-containing species assisted direct growth of graphene ${ }^{64,81,82}$.

(a) Schematic of water-assisted CVD growth of graphene on $\mathrm{SiO}_{2} / \mathrm{Si}$ substrate; (b) and (c) $\mathrm{AFM}$ images of graphene grown on $\mathrm{SiO} / 2 / \mathrm{Si}$ substrates with and without water. Scale bars: $500 \mathrm{~nm}$; (d) Arrhenius plots of grain size versus growth temperature; (e) Schematic illustration of graphene growth on glass with $\mathrm{H}_{2} \mathrm{O}$ assistance; (f) Time-dependent morphology evolution of graphene growth w- $\mathrm{H}_{2} \mathrm{O}$ promoters on quartz glass with growth time of 3, 4, and $5 \mathrm{~h}$, respectively; (g-i) SEM images of graphene samples with uniform shape and size grown by the precursor-modification strategy; (j) Formation energies of the four hybrid structures consisting of graphene nanoribbons deposited on Si-terminated silica. (a-d) Adapted from Elsevier publisher; $(\mathrm{e}, \mathrm{f})$ Adapted from Wiley-VCH publisher; $(\mathrm{g}-\mathrm{j})$ Adapted from American Chemical Society publisher. 
(a)

(d)
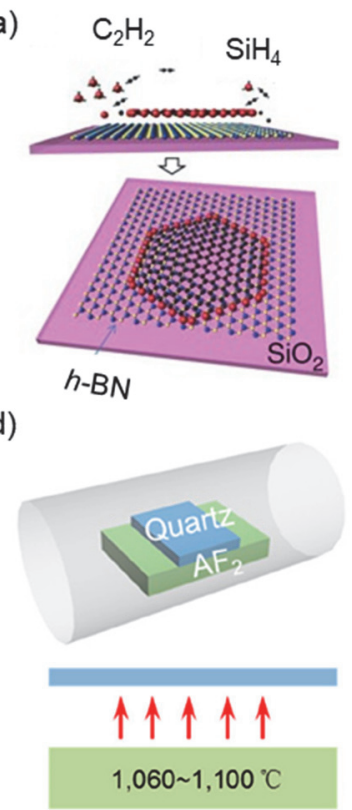

(b)

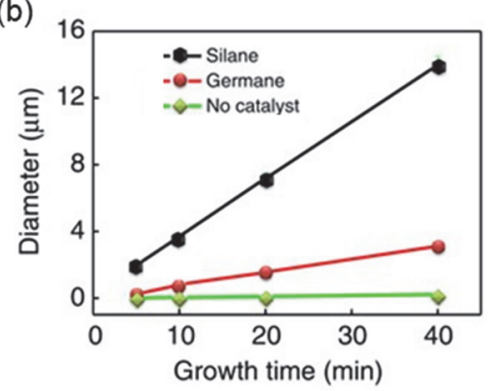

(e)

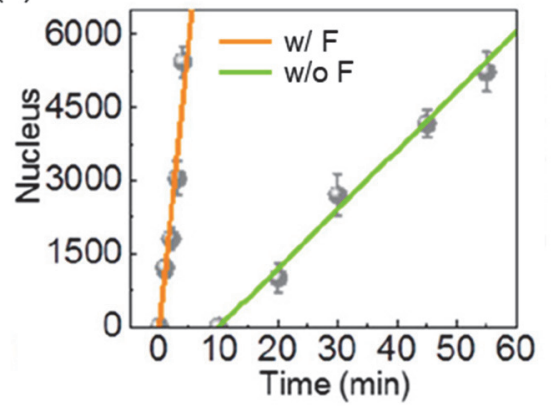

(c)

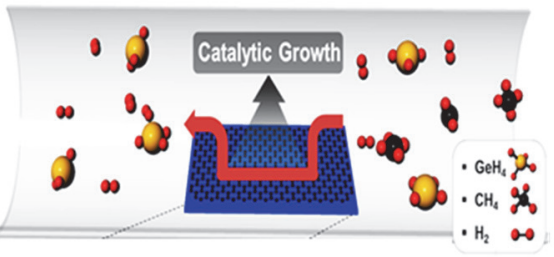

(f)

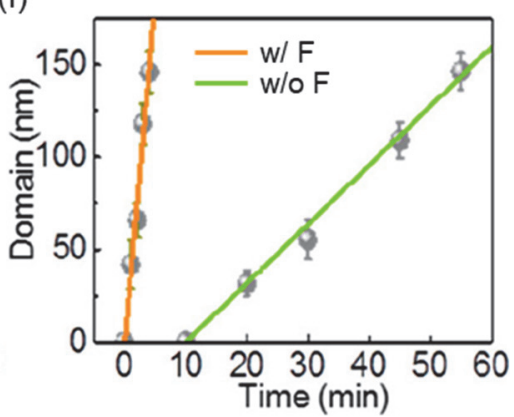

图 6 硅烷/锗烷/氟化钡辅助石墨烯生长 $65,83,84$

Fig. $6 \mathrm{SiH}_{4} / \mathrm{GeH}_{4} / \mathrm{BaF}_{2}$-assisted direct growth of graphene ${ }^{65,83,84}$.

(a) The gaseous catalyst-assisted CVD growth of graphene on h-BN; (b) The growth duration dependence of the domain dimensions for single-crystalline graphene in the presence of silane (black), germane (red) gaseous catalysts and no catalyst (green), respectively; (c) Schematic illustration of gaseous $\mathrm{GeH}_{4}$ catalyst-assisted direct growth of uniform graphene on $\mathrm{SiO}_{2} / \mathrm{Si}$ surface; (d) Diagram of the experimental design and the lateral view of the gap between metal fluoride $\mathrm{AF}_{2}$ and quartz surface; (e) Number of nuclei of graphene as a function of growth time using $\mathrm{BaF}_{2}$ promotor; (f) Time evolution of the domain size by using $\mathrm{BaF}_{2}$ as promotor. (a, b) Adapted from Nature Publishing Group publisher; (c) Adapted from American Institute of Physics publisher; (d-f) Adapted from American Chemical Society publisher.

合能(图5j), 说明甲醇的引入有效避免了石墨烯边 缘与硅原子的强相互作用, 促使石墨烯边缘生长。

\section{3 其他气相化合物}

研究发现, 硅烷、锗烷和氟化物等非金属气相 助剂同样可辅助绝缘衬底上石墨烯的制备(图6a)。 如图6b所示, 硅烷气相助剂可将六方氮化硼祄底 上石墨烯的生长速率和畴区尺寸都提高约两个数 量级, 而锗烷气相助剂也能将生长速率提高近一 个数量级 ${ }^{65}$ 。究其原因在于硅烷裂解产生的硅原子 吸附到石墨烯边缘发挥了催化作用, 有效降低了 活性碳物种拼接到石墨烯边缘形成碳六元环结构 的势垒。进而, 锗烷也用于辅助 $\mathrm{SiO}_{2} / \mathrm{Si}$ 祄底上石墨 烯的成核和生长 (图6c) ${ }^{83}$, 结果表明通过锗烷辅助 法生长的单层石墨烯的载流子迁移率相较于无锗 烷辅助生长法制备的约高一个数量级。同时, 通过 改变锗烷和甲烷的气体含量, 研究者还实现了从 均匀的单层石墨烯到三维石墨烯纳米壁结构的控 制制备。除硅烷、锗烷外, 刘忠范课题组 ${ }^{84}$ 发展了 一种局域供氟法辅助石墨烯快速生长(图6d), 他们 将平整的金属氟化物衬底置于目标石英祄底下 方, 利用其高温释放的氟物种增加了反应体系中
气相活性碳物种浓度, 从而有效提升了石墨烯在 绝缘祄底上的生长速率。如图6e, f所示, 采用局 域供氟法辅助生长时, 石英祄底表面在 $5 \mathrm{~min}$ 内即 可获得满覆盖的石墨烯薄膜; 而普通限域法则需 至少 $60 \mathrm{~min}$ 才能实现, 该方法使石墨烯的生长速率 提高了将近12倍, 成核速率提高了将近9倍。理论 计算表明, 甲烷分子在限域含氟空间内会转化为 活性一氟甲烷分子，该分子在气相中的大量存在 既降低了石墨烯 CVD生长过程中的边缘拼接能 垒, 又可夺去两个近邻生长碳原子中多余的氢原 子, 促进碳六元环形成, 实现石墨烯在绝缘祄底上 快速生长。

\section{5 总结与展望}

本文综述了在绝缘祄底上直接生长石墨烯领 域气相助剂的设计, 系统总结了其相关的策略和 辅助机制。从分析绝缘祄底上石墨烯的生长行为 出发, 介绍了金属与非金属助剂辅助石墨烯生长 的代表性工作。具体而言, 气相助剂辅助法的作用 机理可以分为以下三类: (1)增加反应体系气相中 金属蒸气浓度, 催化提高碳前驱体裂解效率, 促进 
活性碳物种在绝缘祄底上成核及生长。(2)引入氧 气、水、甲醇等含氧物质, 一方面利用其氧化性刻 蚀反应体系中的无定形碳和能量不稳定的石墨烯 核, 从而达到减少无定形碳污染、调控成核密度和 畴区尺寸的目的; 另一方面通过修饰祄底获得羟 基终止表面, 避免石墨烯边缘与祄底的强相互作 用, 从而促进边缘生长, 进而提高石墨烯薄膜的层 数均匀性。(3)向反应体系中引入硅或氟物种, 有 效降低活性碳物种拼接到石墨烯边缘形成碳六元 环结构的势垒, 提高生长速率以实现快速制备。

由于绝缘祄底本身的特殊性, 再加上CVD体 系中石墨烯的生长过程是由发生在祄底表面的反 应和发生在气相中的反应共同调控的 ${ }^{85}$, 使得石墨 烯的直接生长机理十分复杂。迄今为止, 研究者发 展了一系列的生长策略并提出了相应的机制 ${ }^{86-90}$, 但考虑到生长祄底和反应条件的差异, 绝缘祄底 上石墨烯生长的制备科学尚需深入探究。同时, 借 鉴金属祄底上生长石墨烯 ${ }^{91}$ 以及其他二维材料 ${ }^{92}$ 的实践经验, 绝缘祄底自身的粗糙度、洁净度和纯 度等因素也将影响石墨烯的成核及生长行为, 进 一步影响其品质与均匀性等宏观性能。因此, 从祄 底表面预处理角度出发解决石墨烯生长面临的种 种挑战, 或为实现可控制备石墨烯的新途径。此 外, 在实际需求牵引下, 推动材料真正落地的关键 是实现其规模化制备。为发展特定的制备工艺包, 需综合考虑助剂策略的可放大性和生产成本等因 素, 如引入方式、反应室温场和流场的均匀性等。 本文通过梳理气相助剂辅助绝缘祄底上石墨 烯制备的策略, 希望为实现高品质、低成本、规模 化石墨烯直接生长提供思路, 助力早日实现特种 材料上石墨烯生长之 “杀手锏级” 应用。

致谢: 感谢北京大学慈海娜博士、单婧媛博士、姜蓓、袁 吴和陈恒的支持和帮助。

\section{References}

(1) Mayorov, A. S.; Gorbachev, R. V.; Morozov, S. V.; Britnell, L.; Jalil, R.; Ponomarenko, L. A.; Blake, P.; Novoselov, K. S.; Watanabe, K.; Taniguchi, T.; et al. Nano Lett. 2011, 11, 2396. doi: $10.1021 / \mathrm{nl} 200758 \mathrm{~b}$

(2) Zhang, Y. B.; Tan, Y. W.; Stormer, H. L.; Kim, P. Nature 2005, 438, 201. doi: 10.1038/nature04235

(3) Nair, R. R.; Blake, P.; Grigorenko, A. N.; Novoselov, K. S.; Booth, T. J.; Stauber, T.; Peres, N. M.; Geim, A. K. Science 2008, 320 , 1308. doi: $10.1126 /$ science. 1156965

(4) Lee, C.; Wei, X.; Kysar, J. W.; Hone, J. Science 2008, 321, 385. doi: $10.1126 /$ science. 1157996

(5) Balandin, A. A. Nat. Mater. 2011, 10, 569. doi: 10.1038/nmat3064

(6) Dua, V.; Surwade, S. P.; Ammu, S.; Agnihotra, S. R.; Jain, S.; Roberts, K. E.; Park, S.; Ruoff, R. S.; Manohar, S. K. Angew. Chem. Int. Ed. 2010, 122, 2200. doi: 10.1002/ange.200905089

(7) Stoller, M. D.; Park, S.; Zhu, Y.; An, J.; Ruoff, R. S. Nano Lett. 2008, 8, 3498. doi: 10.1021/n1802558y

(8) Goossens, S.; Navickaite, G.; Monasterio, C.; Gupta, S.; Piqueras, J. J.; Perez, R.; Burwell, G.; Nikitskiy, I.; Lasanta, T.; Galan, T.; et al. Nat. Photon. 2017, 11, 366. doi: 10.1038/nphoton.2017.75

(9) Bae, S.; Kim, H.; Lee, Y.; Xu, X.; Park, J. S.; Zheng, Y.; Balakrishnan, J.; Lei, T.; Kim, H. R.; Song, Y. I.; et al. Nat. Nanotechnol. 2010, 5, 574. doi: 10.1038/nnano.2010.132

(10) Romagnoli, M.; Sorianello, V.; Midrio, M.; Koppens, F. H. L.; Huyghebaert, C.; Neumaier, D.; Galli, P.; Templ, W.; D'Errico, A.; Ferrari, A. C. Nat. Rev. Mater. 2018, 3, 392. doi: 10.1038/s41578-018-0040-9

(11) Garaj, S.; Hubbard, W.; Reina, A.; Kong, J.; Branton, D.; Golovchenko, J. A. Nature 2010, 467, 190. doi: 10.1038/nature09379

(12) Xu, M.; Fujita, D.; Hanagata, N. Small 2009, 5, 2638. doi: 10.1002/smll.200900976

(13) Xing, F.; Liu, Z. B.; Deng, Z. C.; Kong, X. T.; Yan, X. Q.; Chen, X. D.; Ye, Q.; Zhang, C. P.; Chen, Y. S.; Tian, J. G. Sci. Rep. 2012, 2, 908. doi: 10.1038/srep00908

(14) Zhao, J.; He, C.; Yang, R.; Shi, Z.; Cheng, M.; Yang, W.; Xie, G.; Wang, D.; Shi, D.; Zhang, G. Appl. Phys. Lett. 2012, 101, 063112. doi: $10.1063 / 1.4742331$

(15) Novoselov, K. S.; Geim, A. K.; Morozov, S. V.; Jiang, D.; Zhang, Y.; Dubonos, S. V.; Grigorieva, I. V.; Firsov, A. A. Science 2004, 306, 666. doi: 10.1126/science. 1102896

(16) Eda, G.; Fanchini, G.; Chhowalla, M. Nat. Nanotechnol. 2008, 3, 270. doi: 10.1038/nnano.2008.83

(17) Hirata, M.; Gotou, T.; Horiuchi, S.; Fujiwara, M.; Ohba, M. Carbon 2004, 42, 2929. doi: 10.1016/j.carbon.2004.07.003

(18) Berger, C.; Song, Z.; Li, X.; Wu, X.; Brown, N.; Naud, C.; Mayou, D.; Li, T.; Hass, J.; Marchenkov, A. N.; et al. Science 2006, 312, 1191. doi: 10.1126/science. 1125925

(19) Li, X.; Cai, W.; An, J.; Kim, S.; Nah, J.; Yang, D.; Piner, R.; Velamakanni, A.; Jung, I.; Tutuc, E.; et al. Science 2009, 324, 1312. doi: $10.1126 /$ science. 1171245

(20) Reina, A.; Jia, X.; Ho, J.; Nezich, D.; Son, H.; Bulovic, V.; Dresselhaus, M. S.; Kong, J. Nano Lett. 2009, 9, 30. doi: $10.1021 / \mathrm{n} 1801827 \mathrm{v}$

(21) Li, X.; Magnuson, C. W.; Venugopal, A.; An, J.; Suk, J. W.; Han, B.; Borysiak, M.; Cai, W.; Velamakanni, A.; Zhu, Y.; et al. Nano 
Lett. 2010, 10, 4328. doi: 10.1021/n1101629g

(22) Wu, T.; Zhang, X.; Yuan, Q.; Xue, J.; Lu, G.; Liu, Z.; Wang, H.; Wang, H.; Ding, F.; Yu, Q.; et al. Nat. Mater. 2016, 15, 43. doi: $10.1038 /$ nmat4477

(23) Lin, L.; Li, J.; Ren, H.; Koh, A. L.; Kang, N.; Peng, H.; Xu, H. Q.; Liu, Z. F. ACS Nano 2016, 10, 2922. doi: $10.1021 /$ acsnano.6b00041

(24) Pang, J.; Mendes, R. G.; Wrobel, P. S.; Wlodarski, M. D.; Ta, H. Q.; Zhao, L.; Giebeler, L.; Trzebicka, B.; Gemming, T.; Fu, L.; et al. ACS Nano 2017, 11, 1946. doi: 10.1021/acsnano.6b08069

(25) Chen, J.; Guo, Y.; Jiang, L.; Xu, Z.; Huang, L.; Xue, Y.; Geng, D.; Wu, B.; Hu, W.; Yu, G.; Liu, Y. Adv. Mater. 2014, 26, 1348. doi: 10.1002/adma.201304872

(26) Chen, Z.; Liu, Z.; Wei, T.; Yang, S.; Dou, Z.; Wang, Y.; Ci, H.; Chang, H.; Qi, Y.; Yan, J.; et al. Adv. Mater. 2019, 31, 1807345. doi: 10.1002/adma.201807345

(27) Chen, Z.; Gao, P.; Liu, Z. F. Acta Phys. -Chim. Sin. 2020, 36, 1907004. [陈召龙, 高鹏, 刘忠范. 物理化学学报, 2020, 36, 1907004.] doi: 10.3866/PKU.WHXB201907004

(28) Yang, W.; Chen, G.; Shi, Z.; Liu, C. C.; Zhang, L.; Xie, G.; Cheng, M.; Wang, D.; Yang, R.; Shi, D.; et al. Nat. Mater. 2013, 12, 792. doi: 10.1038/nmat3695

(29) Chen, L.; Wang, H.; Tang, S.; He, L.; Wang, H. S.; Wang, X.; Xie, H.; Wu, T.; Xia, H.; Li, T.; et al. Nanoscale 2017, 9, 11475. doi: $10.1039 / \mathrm{c} 7 \mathrm{nr} 02578 \mathrm{e}$

(30) Sun, J.; Gao, T.; Song, X.; Zhao, Y.; Lin, Y.; Wang, H.; Ma, D.; Chen, Y.; Xiang, W.; Wang, J.; et al. J. Am. Chem. Soc. 2014, 136, 6574. doi: $10.1021 /$ ja5022602

(31) Chen, J.; Guo, Y.; Wen, Y.; Huang, L.; Xue, Y.; Geng, D.; Wu, B.; Luo, B.; Yu, G.; Liu, Y. Adv. Mater. 2013, 25, 992. doi: 10.1002/adma.201202973

(32) Chen, Z.; Qi, Y.; Chen, X.; Zhang, Y.; Liu, Z. F. Adv. Mater. 2019, 31, 1803639. doi: 10.1002/adma.201803639

(33) Chen, X. D.; Chen, Z.; Jiang, W. S.; Zhang, C.; Sun, J.; Wang, H.; Xin, W.; Lin, L.; Priydarshi, M. K.; Yang, H.; et al. Adv. Mater. 2017, 29, 1603428. doi: 10.1002/adma.201603428

(34) Sun, J.; Chen, Z.; Yuan, L.; Chen, Y.; Ning, J.; Liu, S.; Ma, D.; Song, X.; Priydarshi, M. K.; Bachmatiuk, A.; et al. ACS Nano 2016, 10, 11136. doi: 10.1021/acsnano.6b06066

(35) Sun, J.; Chen, Y.; Priydarshi, M. K.; Chen, Z.; Bachmatiuk, A.; Zou, Z.; Chen, Z.; Song, X.; Gao, Y.; Rummeli, M. H.; et al. Nano Lett. 2015, 15, 5846. doi: 10.1021/acs.nanolett.5b01936

(36) Ruemmeli, M. H.; Bachmatiuk, A.; Scott, A.; Boerrnert, F.; Warner, J. H.; Hoffman, V.; Lin, J.-H.; Cuniberti, G.; Buechner, B. ACS Nano 2010, 4, 4206. doi: 10.1021/nn100971s

(37) Miyasaka, Y.; Nakamura, A.; Temmyo, J. Jpn. J. Appl. Phys. 2011,
50, 04DH12. doi: 10.1143/jjap.50.04dh12

(38) Jiang, B.; Zhao, Q. Y.; Zhang, Z. P.; Liu, B. Z.; Shan, J. Y.; Zhao, L.; Rümmeli, M. H.; Gao, X.; Zhang, Y. F.; Yu, T. J.; et al. Nano Res. 2020, 13, 1564. doi: 10.1007/s12274-020-2771-3

(39) Yan, Z.; Peng, Z.; Sun, Z.; Yao, J.; Zhu, Y.; Liu, Z.; Ajayan, P. M.; Tour, J. M. ACS Nano 2011, 5, 8187. doi: 10.1021/nn202829y

(40) Su, C. Y.; Lu, A. Y.; Wu, C. Y.; Li, Y. T.; Liu, K. K.; Zhang, W.; Lin, S. Y.; Juang, Z. Y.; Zhong, Y. L.; Chen, F. R.; et al. Nano Lett. 2011, 11, 3612. doi: 10.1021/n1201362n

(41) Teng, P. Y.; Lu, C. C.; Akiyama-Hasegawa, K.; Lin, Y. C.; Yeh, C. H.; Suenaga, K.; Chiu, P. W. Nano Lett. 2012, 12, 1379. doi: $10.1021 / \mathrm{nl} 204024 \mathrm{k}$

(42) Murakami, K.; Tanaka, S.; Hirukawa, A.; Hiyama, T.; Kuwajima, T.; Kano, E.; Takeguchi, M.; Fujita, J. I. Appl. Phys. Lett. 2015, 106, 093112. doi: 10.1063/1.4914114

(43) Sun, J. Y.; Chen, Y. B.; Cai, X.; Ma, B. J.; Chen, Z. L.; Priydarshi, M. K.; Chen, K.; Gao, T.; Song, X. J.; Ji, Q. Q.; et al. Nano Res. 2015, 8, 3496. doi: 10.1007/s12274-015-0849-0

(44) Zhang, L. C.; Shi, Z. W.; Wang, Y.; Yang, R.; Shi, D. X.; Zhang, G. Y. Nano Res. 2011, 4, 315. doi: 10.1007/s12274-010-0086-5

(45) Qi, Y.; Deng, B.; Guo, X.; Chen, S.; Gao, J.; Li, T.; Dou, Z.; Ci, H.; Sun, J.; Chen, Z.; et al. Adv. Mater. 2018, 30, 1704839. doi: 10.1002/adma.201704839

(46) Monaghan, S.; Greer, J. C.; Elliott, S. D. J. Appl. Phys. 2005, 97, 114911. doi: 10.1063/1.1926399

(47) Li, X.; Cai, W.; Colombo, L.; Ruoff, R. S. Nano Lett. 2009, 9 , 4268. doi: $10.1021 / \mathrm{n} 1902515 \mathrm{k}$

(48) Lin, L.; Deng, B.; Sun, J.; Peng, H.; Liu, Z. Chem. Rev. 2018, 118, 9281. doi: 10.1021/acs.chemrev.8b00325

(49) Sun, L.; Yuan, G.; Gao, L.; Yang, J.; Chhowalla, M.; Gharahcheshmeh, M. H.; Gleason, K. K.; Choi, Y. S.; Hong, B. H.; Liu, Z. F. Nat. Rev. Methods Primers 2021, 1, 5. doi: 10.1038/s43586-020-00005-y

(50) Fanton, M. A.; Robinson, J. A.; Puls, C.; Liu, Y.; Hollander, M. J.; Weiland, B. E.; Labella, M.; Trumbull, K.; Kasarda, R.; Howsare, C.; et al. ACS Nano 2011, 5, 8062. doi: 10.1021/nn202643t

(51) Chen, X. D.; Chen, Z. L.; Sun, J. Y.; Zhang, Y. F.; Liu, Z. F. Acta Phys. -Chim. Sin. 2016, 32, 14. [陈旭东, 陈召龙, 孙靖宇, 张艳 锋, 刘忠范. 物理化学学报, 2016, 32, 14.] doi: 10.3866/PKU.WHXB201511133

(52) Li, G.; Huang, S. H.; Li, Z. Y. Phys. Chem. Chem. Phys. 2015, 17, 22832. doi: $10.1039 / \mathrm{c} 5 \mathrm{cp} 02301 \mathrm{~g}$

(53) Zhang, J.; Lin, L.; Sun, L.; Huang, Y.; Koh, A. L.; Dang, W.; Yin, J.; Wang, M.; Tan, C.; Li, T.; et al. Adv. Mater. 2017, 29, 1700639. doi: 10.1002/adma.201700639

(54) Lin, L.; Zhang, J.; Su, H.; Li, J.; Sun, L.; Wang, Z.; Xu, F.; Liu, 
C.; Lopatin, S.; Zhu, Y.; et al. Nat. Commun. 2019, 10, 1912. doi: 10.1038/s41467-019-09565-4

(55) Jia, K.; Zhang, J.; Lin, L.; Li, Z.; Gao, J.; Sun, L.; Xue, R.; Li, J.; Kang, N.; Luo, Z.; et al. J. Am. Chem. Soc. 2019, 141, 7670. doi: $10.1021 /$ jacs. 9 b02068

(56) Shan, J.; Fang, S.; Wang, W.; Zhao, W.; Zhang, R.; Liu, B.; Lin, L.; Jiang, B.; Ci, H.; Liu, R.; et al. Natl. Sci. Rev. 2021. doi: $10.1093 /$ nsr/nwab169

(57) Shemella, P.; Nayak, S. K. Appl. Phys. Lett. 2009, 94, 032101. doi: $10.1063 / 1.3070238$

(58) Chen, Z.; Chang, H.; Cheng, T.; Wei, T.; Wang, R.; Yang, S.; Dou, Z.; Liu, B.; Zhang, S.; Xie, Y.; et al. Adv. Funct. Mater.2020, 30, 2070209. doi: 10.1002/adfm.202070209

(59) Kohler, C.; Hajnal, Z.; Deak, P.; Frauenheim, T.; Suhai, S. Phys. Rev. B 2001, 64, 085333. doi: 10.1103/PhysRevB.64.085333

(60) Yazyev, O. V. Phys. Rev. Lett. 2008, 101, 037203. doi: 10.1103/PhysRevLett.101.037203

(61) Hao, Y.; Bharathi, M. S.; Wang, L.; Liu, Y.; Chen, H.; Nie, S.; Wang, X.; Chou, H.; Tan, C.; Fallahazad, B.; et al. Science 2013, 342, 720. doi: 10.1126/science. 1243879

(62) Guo, W.; Jing, F.; Xiao, J.; Zhou, C.; Lin, Y.; Wang, S. Adv. Mater. 2016, 28, 3152. doi: 10.1002/adma.201503705

(63) Chen, J.; Wen, Y.; Guo, Y.; Wu, B.; Huang, L.; Xue, Y.; Geng, D.; Wang, D.; Yu, G.; Liu, Y. J. Am. Chem. Soc. 2011, 133, 17548. doi: $10.1021 / \mathrm{ja} 2063633$

(64) Wei, S.; Ma, L. P.; Chen, M. L.; Liu, Z.; Ma, W.; Sun, D. M.; Cheng, H. M.; Ren, W. Carbon 2019, 148, 241. doi: $10.1016 /$ j.carbon.2019.03.083

(65) Tang, S.; Wang, H.; Wang, H. S.; Sun, Q.; Zhang, X.; Cong, C.; Xie, H.; Liu, X.; Zhou, X.; Huang, F.; et al. Nat. Commun. 2015, 6, 6499. doi: 10.1038/ncomms 7499

(66) Zhu, J.; Xu, H.; Zou, G.; Zhang, W.; Chai, R.; Choi, J.; Wu, J.; Liu, H.; Shen, G.; Fan, H. J. Am. Chem. Soc. 2019, 141, 5392. doi: $10.1021 /$ jacs.9b00047

(67) Peng, Z.; Yan, Z.; Sun, Z.; Tour, J. M. ACS Nano 2011, 5, 8241. doi: $10.1021 / \mathrm{nn} 202923 \mathrm{y}$

(68) Kim, H.; Song, I.; Park, C.; Son, M.; Hong, M.; Kim, Y.; Kim, J. S.; Shin, H. J.; Baik, J.; Choi, H. C. ACS Nano 2013, 7, 6575. doi: $10.1021 / \mathrm{nn} 402847 \mathrm{~W}$

(69) Liu, N.; Zhang, J.; Qiu, Y. F.; Yang, J.; Hu, P. A. Sci. China Chem. 2016, 59, 707. doi: 10.1007/s11426-015-0536-8

(70) Yang, C.; Wu, T. R.; Wang, H. M.; Zhang, X. F.; Shi, Z. Y.; Xie, X. M. Appl. Phys. Lett. 2017, 111, 043107.doi: 10.1063/1.4995559

(71) Zhou, L.; Wei, S.; Ge, C.; Zhao, C.; Guo, B.; Zhang, J.; Zhao, J. Nanomaterials 2019, 9, 964.doi: 10.3390/nano9070964

(72) Song, I.; Park, Y.; Cho, H.; Choi, H. C. Angew. Chem. Int. Ed.
2018, 57, 15374. doi: 10.1002/anie.201805923

(73) Chen, Y. Z.; Medina, H.; Lin, H. C.; Tsai, H. W.; Su, T. Y.; Chueh, Y. L. Nanoscale 2015, 7, 1678. doi: 10.1039/c4nr04627g

(74) Tan, L.; Zeng, M.; Wu, Q.; Chen, L.; Wang, J.; Zhang, T.; Eckert, J.; Rummeli, M. H.; Fu, L. Small 2015, 11, 1840. doi: $10.1002 / \mathrm{smll} .201402427$

(75) Yang, J.; Jiang, Q. Q.; Chen, Z. H.; Hu, P. A.; Li, J. J.; Gu, C. Z.; Yu, G. Diam. Relat. Mater. 2019, 91, 112. doi: 10.1016/j.diamond.2018.11.009

(76) Li, Q. C.; Zhao, Z. F.; Yan, B. M.; Song, X. J.; Zhang, Z. P.; Li, J.; Wu, X. S.; Bian, Z. Q.; Zou, X. L.; Zhang, Y. F.; et al. Adv. Mater. 2017, 29, 1701325. doi: 10.1002/adma.201701325

(77) Zhang, J.; Jia, K.; Lin, L.; Zhao, W.; Quang, H. T.; Sun, L.; Li, T.; Li, Z.; Liu, X.; Zheng, L.; et al. Angew. Chem. Int. Ed. 2019, 58, 14446. doi: 10.1002/anie. 201905672

(78) Zhang, Y. H.; Sui, Y. P.; Chen, Z. Y.; Kang, H.; Li, J.; Wang, S.; Zhao, S. W.; Yu, G. H.; Peng, S. G.; Jin, Z.; Liu, X. Y.; et al. Carbon 2021, 185, 82. doi: 10.1016/j.carbon.2021.09.016

(79) Liu, B. Z.; Wang, H. H.; Gu, W.; Zhou, L.; Chen, Z. L.; Nie, Y. F.; Tan, C. W.; Ci, H. N.; Wei, N.; Cui, L. Z.; et al. Nano Res. 2021, 14, 260. doi: 10.1007/s12274-020-3080-6

(80) Ma, T.; Liu, Z.; Wen, J.; Gao, Y.; Ren, X.; Chen, H.; Jin, C.; Ma, X. L.; Xu, N.; Cheng, H.-M.; et al. Nat. Commun. 2017, 8, 14486. doi: $10.1038 /$ ncomms 14486

(81) Xie, H. H.; Cui, K. J.; Cui, L. Z.; Liu, B. Z.; Yu, Y.; Tan, C. W.; Zhang, Y. Y.; Zhang, Y. F.; Liu, Z. F. Small 2020, 16, 1905485. doi: 10.1002/smll.201905485

(82) Wang, H.; Xue, X.; Jiang, Q.; Wang, Y.; Geng, D.; Cai, L.; Wang, L.; Xu, Z.; Yu, G. J. Am. Chem. Soc. 2019, 141, 11004. doi: $10.1021 /$ jacs.9b05705

(83) Lee, J. H.; Kim, M. S.; Lim, J. Y.; Jung, S. H.; Kang, S. G.; Shin, H. J.; Choi, J. Y.; Hwang, S. W.; Whang, D. Appl. Phys. Lett. 2016, 109, 053102. doi: 10.1063/1.4960293

(84) Xie, Y.; Cheng, T.; Liu, C.; Chen, K.; Cheng, Y.; Chen, Z.; Qiu, L.; Cui, G.; Yu, Y.; Cui, L.; et al. ACS Nano 2019, 13, 10272. doi: 10.1021/acsnano.9b03596

(85) Chen, H.; Zhang, J.; Liu, X.; Liu, Z. F. Acta Phys. -Chim. Sin. 2022, 38, 2101053. [陈恒, 张金灿, 刘晓婷, 刘忠范. 物理化学 学报, 2022, 38, 2101053.] doi: 10.3866/PKU.WHXB202101053

(86) Bachmatiuk, A.; Borrnert, F.; Grobosch, M.; Schaffel, F.; Wolff, U.; Scott, A.; Zaka, M.; Warner, J. H.; Klingeler, R.; Knupfer, M.; et al. ACS Nano 2009, 3, 4098. doi: 10.1021/nn9009278

(87) Hong, G.; Wu, Q.-H.; Ren, J.; Lee, S. T. Appl. Phys. Lett 2012, 100, 231604. doi: $10.1063 / 1.4726114$

(88) Sun, J.; Zhang, Y.; Liu, Z. F. ChemNanoMat 2016, $2,9$. doi: $10.1002 / \mathrm{cnma} .201500160$ 
(89) Saito, K.; Ogino, T. J. Phys. Chem. C 2014, 118, 5523. doi: $10.1021 / \mathrm{jp} 408126 \mathrm{e}$

(90) Medina, H.; Lin, Y. C.; Jin, C.; Lu, C. C.; Yeh, C. H.; Huang, K. P.; Suenaga, K.; Robertson, J.; Chiu, P. W. Adv. Funct. Mater. 2012, 22, 2123. doi: 10.1002/adfm.201102423

(91) Cheng, T.; Sun, L.; Liu, Z.; Ding, F.; Liu, Z. F. Acta Phys. -Chim. Sin. 2022, 38, 2012006. [程婷, 孙禄钊, 刘志荣, 丁峰, 刘忠范.
物理化学学报, 2022, 38, 2012006.]

doi: 10.3866/PKU.WHXB202012006

(92) Chang, C.; Chen, W.; Chen, Y.; Chen, Y.; Chen, Y.; Ding, F.; Fan, C.; Jin Fan, H.; Fan, Z.; Gong, C.; et al. Acta Phys. -Chim. Sin. 2021, 37, 2108017. [常诚, 陈伟, 陈也, 陈永华, 陈雨, 丁峰, 柇春海, 范红金, 范战西, 龚成, 等. 物理化学学报, 2021, 37, 2108017.] doi: 10.3866/PKU.WHXB202108017 